\title{
http://dx.doi.org/10.35381/racji.v5i1.599
}

\section{El derecho a la adopción de parejas del mismo sexo: El caso ecuatoriano}

The right to adoption of couples of the same sex: The ecuadorian case

\author{
Silvia Elizabeth Torres-Pérez \\ silvia.torres@psg.ucacue.edu.ec \\ Universidad Católica de Cuenca, Cuenca \\ Ecuador \\ https://orcid.org/0000-0002-3335-5321 \\ Cecilia Ivonne Narváez-Zurita \\ inarvaez@ucacue.edu.ec \\ Universidad Católica de Cuenca, Cuenca \\ Ecuador \\ https://orcid.org/0000-0002-7437-9880 \\ Camilo Emanuel Pinos-Jaén \\ cpinosj@ucacue.edu.ec \\ Universidad Católica de Cuenca, Cuenca \\ Ecuador \\ https://orcid.org/0000-0002-0934-8471 \\ Juan Carlos Erazo-Álvarez \\ jcerazo@ucacue.edu.ec \\ Universidad Católica de Cuenca, Cuenca \\ Ecuador \\ https://orcid.org/0000-0001-6480-2270
}

Recibido: 14 de noviembre de 2019

Aprobado: 15 de diciembre de 2019

\section{RESUMEN}

La adopción para parejas del mismo sexo, sigue siendo una prohibición que consta dentro de la Constitución de la República del Ecuador aprobada en el año 2008 y ha sido analizado desde las posiciones sociales y jurídicas, así como la visión y crítica que al tema se han generado a nivel nacional e internacional. La metodología científica investigativa parte del paradigma cualitativo, sustentado en métodos como análisis y 
síntesis bibliográficos, método histórico-lógico; la recolección de la información de las posturas se ha realizado a través de la revisión bibliográfica accediendo a textos, normas y artículos ubicados en repositorios científicos autorizados; los datos han sido sintetizados y presentados de manera adecuada. Se concluye que el derecho a la adopción en parejas del mismo sexo en el Ecuador continúa siendo negado, lo que es considerado como una vulneración hacia los derechos de la niñez.

Descriptores: Adopción; Familia; Homoparentalidad; Derechos; Vulneración.

\begin{abstract}
The adoption for same-sex couples, remains a prohibition that appears within the Constitution of the Republic of Ecuador approved in 2008 and has been analyzed from the social and legal positions, as well as the vision and criticism that the subject has been generated nationally and internationally. The scientific research methodology starts from the qualitative paradigm, based on methods such as bibliographic analysis and synthesis, historical-logical method; the collection of the information of the positions has been carried out through the bibliographic revision accessing texts, norms and articles located in authorized scientific repositories; The data has been synthesized and presented in an appropriate manner. It is concluded that the right to adoption in samesex couples in Ecuador continues to be denied, which is considered a violation of the rights of children.
\end{abstract}

Descriptors: Adoption; Family; Homoparentality; Rights; Violation.

\title{
INTRODUCCIÓN
}

En Ecuador, el tema de la adopción como un derecho concedido a parejas consideradas como no heterosexuales está todavía en discusión. Las posiciones diversas desde grupos sociales que defienden la conformación de familias tradicionales e incluso, la posición de las iglesias, generan duros y severos criterios hacia la concesión de derechos a la comunidad de Lesbianas, Gays, Bisexuales, Transexuales e Intersexuales (LGBTI). Es evidente que persiste el estigma y la discriminación hacia las familias conformadas por parejas del mismo sexo, cuya orientación sexual es vista como uno de los principales factores para este fenómeno; muy a pesar de que a nivel 
mundial las personas de la comunidad LGBTI, han alcanzado logros en materia de derechos muy importantes como es el matrimonio igualitario (Véliz Valencia, 2019). Un logro fundamental es la evidencia científica que avala la posición de que la orientación sexual de los padres no tiene influencia dentro del proceso de desarrollo de los niños, niñas y adolescentes; e incluso no incide en la elección de la vida sexual y reproductiva. La crianza o educación que reciban los niños adoptados por parejas del mismo sexo no es un factor nocivo o negativo en las pautas comportamentales que asuman conforme van creciendo (Campos y Herazo, 2015).

En el Ecuador la Constitución de la República establece desde el principio el respeto de los derechos de todos los ciudadanos, sin que su orientación sexual sea motivo de discriminación bajo ninguna circunstancia. El artículo 67 de la Constitución de la República aprobada en el año 2008, claramente se especifica que "se reconoce la familia en sus diversos tipos" (Asamblea Nacional, 2008, p. 50), por lo que la variante surgida por la igualdad de derechos de las personas es un tipo de familia que debe ser protegida por los Estados y les deben ser reconocidos aquellos derechos que les son inherentes desde su misma base. Este reconocimiento es importante para sustentar la teoría del derecho a la adopción y evitar que se vulneren los derechos de igualdad y no discriminación de las parejas no heterosexuales, ya que asumen un compromiso de formar un hogar y brindar seguridad emocional a un niño, niña o adolescente que lo necesite.

Lo antes expuesto, deja en evidencia la problemática identificada en Ecuador, ya que a pesar del reconocimiento que a nivel mundial y nacional se ha hecho sobre los derechos a las personas LGBTI, incluido el matrimonio igualitario, aún faltan muchos otros derechos por ser aceptados. En el Ecuador en el año 2019 se dio por parte de la Corte Constitucional la sentencia mediante la cual se hace el reconocimiento del matrimonio en parejas del mismo sexo como un derecho que permite la igualdad real entre todas las personas. El derecho a adoptar a estas familias sigue siéndoles negados desde la misma Constitución de la República, imposibilitándoles formar una 
familia y brindar a un niño, niña o adolescente el acceso a las atenciones de un hogar. El presente estudio tiene como objetivo analizar el derecho a la adopción de parejas del mismo sexo desde el caso ecuatoriano, evidenciando la vulneración de los derechos de igualdad y no discriminación a la que se ven sometidos las parejas no heterosexuales.

\section{DESARROLLO}

\section{El Derecho Constitucional ecuatoriano y el reconocimiento de las familias homoparentales}

La familia de acuerdo con Bowen (1991) puede ser definida como el sistema en el cual sus miembros pretenden que los demás miembros que la conforman encuentren estabilidad basada en la dinámica normal de interrelación, garantizando de esta forma la funcionalidad y el establecimiento de su identidad, dando además la seguridad sobre aspectos como son la afectividad, la emocionalidad, la satisfacción de necesidades mentales, psicológicas que se encaminan en obtener el desarrollo integral de toda persona.

Otra definición presenta Rodríguez (2012) sustentando que la familia es una institución que desde los orígenes de la humanidad y de forma natural ha ido creando en su interior normas, leyes, criterios éticos y morales, así como la disciplina hacia todos quienes la conforman, siendo encabezada por su miembro más antiguo, más sabio o con experiencia, que guía a los otros hacia los rituales o tradiciones que mantienen.

Continuando con la revisión de las definiciones de la familia, se observa lo que manifiesta Hernández (1998), para quien este grupo humano viene a representar la interacción en la vida cotidiana como un agente dentro de la sociedad y que cumple un papel fundamental dentro de la estructura de la misma y que por lo tanto incide dentro de otras instituciones sociales, ya sea de forma activa o pasiva. La familia es la primera organización institucional, en la cual se considera que el ser humano desde que nace empieza a aprender las normas para desempeñarse dentro de la sociedad en general, el ser humano crece y se desarrolla bajo el sistema familiar que le aporta a la vez 
características de su personalidad, viviendo bajo un sistema de autoridad distribuido entre los miembros de más alto rango que cumplen roles y funciones de forma integral y a la vez interdependientes (Aylwin y Solar, 2012).

La Constitución de la República del Ecuador señala en su artículo 67 que el Estado ecuatoriano reconoce todos los tipos de familia, garantizando su protección y la implementación de programas y condiciones necesarias para que su desarrollo sea integral, además del bienestar de todos sus miembros. La familia puede ser conocida desde diversos puntos, siendo diferenciada por características desde los puntos de vista de profesionales como los sociólogos, psicólogos.

Un concepto sobre lo que es la familia se encuentra en la Declaración Universal de los Derechos Humanos que señala: "es el elemento natural y fundamental de la sociedad y tiene derecho a la protección de la sociedad y del Estado" (ONU, 1948, p. 2).

Dentro del sistema de familia también existen subsistemas como el de familia nuclear: "padres e hijos; también se conoce como círculo familiar" (Zambrano, 2017, p. 2). La Constitución del Ecuador en su artículo 1, hace mención a que este país sigue la línea constitucional haciendo énfasis en la garantía y defensa de los derechos y justicia a todos sus habitantes; de tal forma que no se trata de una mera referencia, sino que hace implícita la responsabilidad que adquiere el país como garantista de los derechos de los seres humanos en términos de igualdad y sin discriminación alguna.

Entre estos derechos se encuentra la garantía a la conformación de la familia, bajo un concepto que en la actualidad se ha vuelto diverso, producto de la ampliación de las formas de constitución de las mismas. La diversidad dentro de las familias es uno de los puntos que en la actualidad ha generado muchos criterios contrarios y a favor, sobre todo en cuanto al nuevo tipo de familia: homoparentales (Romo, 2013). Este tipo de familias se definen como aquellas "constituidas por personas del mismo sexo que pueden cumplir el rol de padres o madres" (Ruiz, 2019, p. 12), estos padres llegan a cumplir sus fines al crear lazos de filiación con hijos adoptados, conseguidos a través de un vientre de alquiler o de inseminación artificial. De esta forma el concepto de 
familia queda establecido como el sistema en que se conforma un hogar entre parejas del mismo sexo. Por el contrario, otra definición la brinda Agrest (2007) quien afirma que las parejas no heterosexuales son una familia homoparental sin necesidad de tener hijos.

En el caso ecuatoriano, la Corte Constitucional hizo el reconocimiento de las familias homoparentales mediante la Sentencia No. 184-18-SEP-CC, siendo emitida como respuesta a la demanda presentada por Nicola Susan Rothon y Helen Louis Bicknel para el reconocimiento de los hijos nacidos dentro de su unión legalizada tanto en el Ecuador como en Reino Unido país del cual son natales, ya que ambas pertenecen a la comunidad LGBTI. Para asumir el rol de madres debieron superar las barreras que sobre el nuevo tipo de familia había en el país, por lo que interpusieron una demanda en búsqueda de lograr la inscripción de sus hijos con sus apellidos (Caso BicknellRothon en contra del Estado ecuatoriano, 2018).

Se niega la inscripción ya que en el país no existía la normativa que permitía inscribir a un menor con los apellidos de dos madres o dos padres. Ante esta realidad las señoras interponen el debido recurso en busca de una solución que les permitiera a sus hijos acceder al derecho a la identidad. En primera instancia la demanda es negada y ante la interposición de una acción de protección se ratifica la misma. Se presenta enseguida la Acción Ordinaria de Protección ante la Corte Constitucional, la cual acepta el reconocimiento de las familias homoparentales.

Es importante en este punto hacer las consideraciones sobre la protección que a la Familia se le brinda a nivel internacional, considerando que no se trata de un concepto aparte o separado, sino que se trata de un aspecto universalista y progresista. Entre las organizaciones que brinda protección se encuentra la Organización de Naciones Unidas (ONU), que en todos los instrumentos que se han emitido aunque no se la nombre directamente, se entiende que es la familia la beneficiada ya que se atiende a las necesidades y la satisfacción de las mismas a todos a todos sus miembros, de forma 
especial a los considerados vulnerables como los niños, ancianos, mujeres o personas con discapacidad (Sánz, 2013).

La ONU ha evitado emitir un concepto propio sobre lo que es la familia, sino que por el contrario está abierta a aceptar todo tipo de formas de conformación de las mismas, sin atender a la forma de su estructura tradicional como por ejemplo las internacionales, uniparentales, ampliadas, entre otras. Por ello, la protección que le otorgan se enfoca precisamente en que esa estructura sea estable y posea sentido de bienestar, sin prejuzgamientos contra la protección que debe dárseles. Organismos dentro de la ONU como lo es la UNICEF si han dado su punto de vista, atendiendo a que es cambiante y por lo tanto diversa, que varía acorde a las condiciones sociales, económicas, políticas e históricas, enfatizando que cualquier sea su estructura, es el núcleo que sostiene todo sistema social en el cual se entretejen las relaciones personales e interpersonales (lbídem).

La familia a pesar de ser considerada una institución establecida desde los mismos inicios de la humanidad, no ha visto disminuir la fortaleza de su conformación, por el contrario, las relaciones interpersonales de sus miembros han sido motivo para elevarla como una comunidad de afecto estable, ordenado, cambiante y diverso. Los gobiernos han incluido en sus normas estipulaciones precisas para garantizar su protección, la misma que a nivel internacional es de carácter importante.

\section{La adopción por parte de parejas de mismo sexo}

La palabra adopción proviene del término latín adoptio que podría ser interpretado como profijamiento, que en términos de filiación es la pertenencia de una persona a otra, considerando esa unión de acuerdo a la ley y no conforme lo puede dictar la naturaleza, como es el caso de los lazos biológicos (Durán, 2012). La adopción es la creación de un lazo de filiación entre un padre e hijo que no comparten ningún tipo de relación de consanguinidad. En la edad antigua, pueblos como Babilonia, Israel, Egipto, 
Atenas, India, Irán, Roma hacían uso de esta figura como parte de sus normas y costumbres (Durán, 2012).

En la edad media, la adopción va perdiendo sentido en su aplicabilidad, sobre todo cuando luego de realizada la misma, las parejas llegaban a tener hijos biológicos propios, es así como en España y Francia surgen las figuras de adrogación y perfeccionan el contrato para unir a través de la misma a las familias nobles, sin riqueza con miembros de familias ricas, sin sangre noble. Avanzando en la historia se llega a la edad moderna, en este sentido, la adopción alcanza su máxima expresión como un medio de solucionar los problemas sociales que resultaron de los hogares destruidos, padres sin hijos e hijos sin padres, huérfanos de las guerras. Desde este momento la adopción llega a ser reconocida como la respuesta para solucionar las deficiencias de la infancia sin hogar.

En este sentido, González (2015) menciona que, al ser una oportunidad legalmente válida para conformar una familia, la adopción facilita la creación de lazos filiales, aunque no biológicos que garantizan la protección ante la situación de desamparo que pudieran estar viviendo los menores. El mismo autor hace mención a la importancia social que tiene la adopción, ya que otra de sus finalidades es la de que el proceso garantice que será por el bienestar e interés superior del menor, como el principio máximo para que la figura no sea considerada un negocio, sino por el contrario, sea relevante el bienestar del menor.

Para las familias conformadas por padres del mismo sexo, la adopción es un derecho que implica directamente la creación de un escenario familiar que no dista mucho de uno conformado por padres heterosexuales. La orientación sexual no implica que genere daños negativos en el desarrollo infantil, sino que los valores y principios no interfieren en su desarrollo afectivo, psicológico e incluso social, aunque estas familias están en una constante vigilancia para demostrar su aptitud para la crianza de sus hijos (Placeres, Olver, Rosero, y Urgilés, 2017). 
Los principios de la adopción en Ecuador se encuentran establecidos en el art. 153 del Código de la Niñez y Adolescencia (2012), que de forma resumida especifica que esta figura será aplicada única y exclusivamente como una forma de ayuda en los casos cuando hayan fracasado las otras medidas para garantizar la reunificación familiar, dando prioridad a la adopción nacional, facilitando la internacional, en casos expresos y determinados como excepcionales.

Entre los principios de la adopción resalta el texto constante dentro del art. 153, numeral tercero, en el cual se dice textualmente: "3.- Se priorizará la adopción por parte de parejas heterosexuales constituidas legalmente, sobre la adopción por parte de personas solas" (Asamblea Nacional, 2012, p. 59). Es evidente dentro de este numeral que se sigue manteniendo la disposición de la Constitución de la República, que en el último inciso del artículo 68 claramente ordena que "la adopción corresponderá sólo a parejas de distinto sexo" (Asamblea Nacional, 2008, pág. 51), impidiendo de esta manera que personas de distinto sexo accedan a la conformación de un hogar con hijos o hijas.

Actualmente las sociedades se enfrentan a cambios de sus estructuras, en las que se requiere aceptar que las personas ya no son sólo de género/sexo masculino o femenino, sino que la diversidad en razón de la orientación sexual es un tema que sustenta las modificaciones a normas, leyes o códigos de Estados.

En el año 2008, con la vigencia de la Constitución de la República del Ecuador, el movimiento LGBTI vivió momentos de tensión con la imposición de la negativa de adopción a personas de distinto sexo, enfatizando que solamente las parejas heterosexuales son consideradas como normales para poder acceder a la adopción y conformación de una familia. La imposibilidad de acceder al matrimonio fue otro revés al reconocimiento de la igualdad de sus derechos, aunque con el tiempo consiguieron que las uniones de hecho fueran formalizadas y consideradas con los mismos derechos que las uniones de hecho heterosexuales. 
A pesar de ello, sigue existiendo la negativa de que puedan acceder a la adopción de forma legal, a pesar de que a nivel mundial y en la región sudamericana, países vecinos hayan aceptado reformar sus legislaciones internas para facilitar no sólo el matrimonio homosexual, sino también brindarles la oportunidad de adoptar niños, tal es el caso de Uruguay y Colombia. Estos países han sustentado sus decisiones en razón de hacer válido el principio de igualdad para todas las personas y de no discriminación por la diversidad en su orientación sexual. Además de ello, se han emitido investigaciones científicas cuyos resultados favorecen la adopción de niños por parejas del mismo sexo, negando las posiciones que los descalifican como padres ente la posibilidad de que sufran efectos psicológicos nocivos, afecten su desarrollo integral e incluso sean motivo para confundirlos en su sexualidad.

Al respecto, las reformas enfatizan lo dispuesto en dos instrumentos internacionales importantes: a) la Declaración de los Derechos del Niño, Art. 6 que menciona que todo menor de edad tiene derecho a vivir bajo el "amparo y responsabilidad de sus padres, en un ambiente de afecto y de seguridad moral y material; no se debe separar al niño de corta edad de su madre" (Gómez, Berástegui y Adroher, 2015, p. 22); b) Art. 3 "Declaración sobre los Principios Sociales y Jurídicos relativos a la protección y el bienestar de los niños, con especial referencia a la adopción y la colocación en hogares de guarda, en los planos nacional e internacional, recoge como primera prioridad que el niño sea cuidado por sus padres" (Ibídem, p. 22).

Como se ha mencionado con anterioridad en esta investigación, el art. 153 del Código de la Niñez y Adolescencia, numeral 3 es claro al especificar que sólo será posible acceder a la adopción a parejas heterosexuales "constituidas legalmente, sobre la adopción por parte de personas solas" (Asamblea Nacional, 2012, p. 59). Al analizar la normativa internacional como nacional es evidente que no se le da la posibilidad de adoptar a las parejas del mismo sexo, sino sólo a las heterosexuales, manteniendo de esta forma una barrera que imposibilita a estas parejas a acceder al derecho de adopción (Bolaños, Colorado, Quintero, y Mesa, 2019). 
Es conocido que las familias en su conformación inicial han sufrido cambios, por lo que una de las formas de las mismas es la homoparental, establecida cuando los padres son del mismo sexo, acudiendo a la adopción para crear un hogar, "en la actualidad donde se vive una intensa aceleración a partir del reconocimiento legal del matrimonio entre personas del mismo sexo en muchas naciones" (Placeres, Olver, Rosero, y Urgilés , 2017, p. 361)

En la República de Colombia, la figura de la adopción como un derecho cedido a parejas del mismo sexo antes de las reformas que permitieron su reivindicación, generaron en el país una serie de discusiones que dividieron la opinión de la sociedad, posicionándose tanto a favor como en contra. Aquellos que se niegan a que este derecho les haya sido concedido a las personas LGBTI sostienen que esto implica confusiones para los niños que sean criados por padres no heterosexuales (Rengifo, 2016).

La figura de la adopción fue creada con una finalidad muy enfática y precisa: brindar una familia o un hogar a los niños, niñas o adolescentes que carecen de la misma, que ven en las parejas solicitantes una oportunidad de poder acceder a una y de recibir la atención, el cariño y la estabilidad emocional que necesitan para su normal y completo desarrollo. Es indiscutible que este posicionamiento de rechazo hacia la conformación de un nuevo tipo de familia está enraizado por motivos homofóbicos, así como la enraizada idea de que la familia debe estar conformada por el padre, madre e hijos.

\section{Vulneración de los derechos de igualdad y no discriminación a parejas no heterosexuales en el Ecuador}

La diferencia en la orientación sexual es el punto focal que da origen a la homofobia y la estigmatización desde inicios del siglo XIX. Los movimientos que surgieron en esta época se caracterizaron por el liberalismo en el que se vivía la vida privada, la naturalidad en la cual la sexualidad individual iba siendo asumida y la eliminación de castigos para quienes no eran heterosexuales (Ramírez y Lozada, 2017). 
Finalizada la segunda guerra mundial y en los años posteriores, los cambios surgidos en el feminismo, el pacifismo con el amor libre y el reconocimiento de los afroamericanos dieron paso al afianzamiento del Movimiento Gay que en Estados Unidos se desarrollaba con cierta falta de respeto hacia las normas civiles, sin embargo, poco a poco fueron acabando con los clichés hacia los grupos de personas homosexuales, los cuales empezaron a incrementar sus demandas para que se les reconozcan la igualdad de los derechos y el cese de las persecuciones a las que se veían sometidos.

Para la década de los años 80 el hallazgo del VIH-SIDA, como lo mencionan Ramírez y Lozada (2017), fue considerada una enfermedad asociada al homosexualismo, debido en gran parte a que sus primeras víctimas pertenecían a este movimiento o habían declarado públicamente su orientación sexual, lo que fue el punto de partida para aumentar la discriminación y posicionar la heterosexualidad como un sistema sexual normal. Para los años 90 el movimiento LGBT (estas eran sus siglas iniciales) fue creciendo en la integración social, se llegó incluso a aceptar con cierto grado de normalidad el que hombres homosexuales sean aceptados en las filas del Ejército de los Estados Unidos, e incluso en un país tan radical religiosamente como lo es Israel.

El avance de la tecnología en esta época ha favorecido la reivindicación de los derechos de la comunidad LGBTI. La diversidad sexual fue incluida en los Principios de Yogyakarta en el año 2007, para enfatizar que el Derecho Internacional reconoció sus ideales y los continuos pedidos de igualdad en Derechos Humanos, así como el sentir de la falta del matrimonio igualitario, la emisión de leyes antidiscriminatorias y la adopción a parejas del mismo sexo.

Estos principios reconocen internacionalmente los Derechos Humanos de las personas de orientación sexual diversa o LGBTI. En el año 2006 se reunieron un grupo de expertos de 25 países en materia de identidad de género en Indonesia. Como tal estos principios entraron en vigencia desde su publicación desde marzo del año 2007 en la ONU (ONU, 2007). La redacción de los principios sigue el esquema de un tratado 
internacional en esta materia, aunque enfocados en el respeto de la igualdad de las personas LGBTI, lo que es un avance significativo para este grupo de personas considerados como vulnerables y en minoría. Además de la opinión de los expertos, el instrumento recoge las recomendaciones de la comunidad internacional que aboga para la prevención de toda forma de discriminación (Pulecio, 2015).

El instrumento se enfoca en demostrar la poca importancia que se le ha dado al tema de la diversidad por orientación sexual y la defensa de sus derechos; así como hace mención a las violaciones, discriminaciones y estigmatizaciones que sufren las personas que se engloban en esta diversidad. Como un punto focal hace mención al caso concreto de Uganda, país en el cual la discusión sobre el reconocimiento de estos derechos ha dado paso a una ola creciente de actos violentos.

En el plano internacional los Principios de Yogyakarta muestran que existe un continuo irrespeto por el derecho a la vida e integridad física de las personas no heterosexuales, siendo víctimas de persecuciones y prejuicios. La parte del preámbulo de estos principios, hace la exposición de los motivos que dieron paso a la promulgación del instrumento; a continuación, se presentan las definiciones sobre orientación sexual e identidad de género son utilizados a nivel internacional. Estas definiciones se centran en la amplitud de lo que es sexo/género, no concentrada en sólo hombre y mujer, sino en dar a conocer la sexualidad humana en su diversidad. Particularmente el principio 3 se enfocan en garantizar que los Estados Parte reconozcan los derechos de las personas no heterosexuales desde su personalidad jurídica, hasta el poder contraer matrimonio y formar una familia que es el objetivo del principio 24 (ONU, 2007).

El documento tiene la calidad jurídica de ser fuente del Derecho Internacional, por lo que su cumplimiento como norma es fundamental en el tema de la diversidad sexual. La Comisión Interamericana de Derechos Humanos (CIDH) en el año 2015 emite el informe sobre la violencia a las personas LGBTI en América, documentando de esta forma los casos de violencia que en forma generalizada se han dado y se siguen dando por motivos de género a este colectivo, que incluso está sobrepasando las estadísticas 
de la violencia de género hacia las mujeres. Es realmente preocupante conocer las formas de violencia que se aplican a las personas LGBTI, el reconocimiento de sus derechos sigue avanzando, no obstante, parece que las formas de violencia no están disminuyendo.

El informe publicado por la CIDH es considerado como el primero en su clase, ya que abarca aspectos sistemáticos en cuanto a lo que es la violencia a personas LGBTI. Enfatiza el informe que las sociedades americanas siguen el esquema de heteronormatividad en cuanto a dos grupos de género y sexo: hombre y mujer; persiste la intolerancia hacia quienes se salen de este esquema, formas de irrespeto y discriminación son la constante con la que se encuentran en diversos ámbitos como son el laboral, social, educativo, etc. Por ello, el informe determina que las formas de violencia más comunes desde diversos ámbitos son: físicos, social, prejuicio, correctiva y médica. Cada una de las mencionadas tiene formas de actuación distintas, a saber:

a) Física: evidenciada con golpes, de forma cruel y violenta, que cusa heridas o lesiones temporales o permanentes e incluso la muerte.

b) Social: que nace de la violencia física y que se evidencia en la discriminación ante los cambios corporales físicos, por lo que no son considerados parte de grupos sociales tradicionales.

c) Prejuicio: tal como lo indica la $\mathrm{CIDH}$ en su informe, se trata de expresiones no racionalizadas hacia las personas con diversidad en su orientación sexual, que aumenta ante la complicidad de la sociedad.

d) Criminal: que a su vez es derivada de la anterior y efecto de la primera, donde la violencia es sólo motivada por no ser heterosexuales.

e) Correctiva: quizás la más cruel de todas las formas, debido la violencia de actos sexuales como norma de corrección perversa para que las personas encaucen su vida sexual. 
f) Médica: considerada como aquella que es cometida desde los servicios de salud públicos o privados, en actos como uso de lenguaje ofensivo o burlesco, exclusión en la atención o interposición de barreras (Quinche, 2016).

La familia tal como lo afirman Palacio y Cárdenas (2017) ha sido asociada con aquella conformada por parejas del mismo sexo, con el sentido de procrear hijos, a quienes deben desde el primer momento brindarles su protección, cuidados, educación, etc.

En los años previos a la finalización del siglo XX y comienzos del nuevo siglo, el posicionamiento en aumento de los movimientos sociales, de forma especial de las poblaciones como las LGTBI, quienes han luchado por el reconocimiento de sus derechos en igualdad de condiciones y últimamente el de adopción. La diversidad de las familias en la actualidad, implica que no sólo sean formados por personas de distinto sexo, sino también aquellas parejas del mismo sexo.

\section{METODOLOGÍA}

La metodología empleada en la construcción de este estudio, se sustentó en el enfoque cualitativo, el cual posibilitó la recolección de información enfocada en el tema y problema planteado en esta investigación a partir de posiciones culturales e ideológicas distintas sobre las parejas del mismo sexo, la concesión de derechos como el matrimonio igualitario y el acceso a la adopción, así como las formas de violencia a la que se han visto sometidos. Entre los métodos utilizados están el histórico-lógico con el análisis de la historia de la familia y evolución de los derechos a las personas de la comunidad LGBTI para conocer cómo se han desarrollado a través de la historia de la humanidad y de qué forma han sido reconocidos esos cambios; la principal técnica de investigación utilizada fue la revisión bibliográfica mediante el acceso a leyes, artículos, textos, procesos judiciales y otros documentos jurídicos de autores nacionales y extranjeros, los que fueron analizados y sintetizados reflexivamente para la construcción teórica (Ruiz, Narváez y Erazo, 2019). 


\section{APORTES GENERADOS}

Las familias son consideradas desde las etapas tempranas de la humanidad como la base fundamental de toda sociedad. Las formas de familias tradicionales se han centrado siempre en el modelo tradicional: padre, madre e hijos. Este modelo no ha cambiado hasta entrar a nuevas versiones de la familia, habiéndose ampliado la misma con el reconocimiento de otro tipo de familias como son las homoparentales, conformadas por padres del mismo sexo, en las que se asume que sus integrantes a su vez gozan de derechos y deberes, así como de todo tipo de garantías que beneficien su desarrollo.

La Constitución de la República del Ecuador, especifica en su artículo 67 sobre el reconocimiento y protección a la familia en todas sus formas, incluso debiendo establecer límites a su poder estatal ya que no puede atentar contra la misma. Dentro de estas garantías se encuentra la del respeto a la diversidad por motivos de orientación sexual. En el plano internacional la ONU ha demostrado la defensa sobre la familia, pero sin emitir una definición concisa sobre la misma, haciendo de esta forma la aceptación de que existen formas distintas de conformación de las mismas.

A pesar del reconocimiento logrado a nivel internacional con los principios, la violencia que se ejerce en contra de las personas no heterosexuales es aún constante. Las formas de violencia a la que se ven sometidos ha llegado a alcanzar niveles de crueldad e inhumanidad, por la forma en la cual sufren vejaciones físicas, psicológicas, morales que incluso llegan a la muerte.

Se ha visto y confirmado que la adopción de niños por parejas del mismo sexo no causan daños psicológicos o influyen en la elección de la orientación sexual de los menores, sino que por el contrario y como el caso del vecino país Colombia, se ha comprobado científicamente que los niños, niñas y adolescentes que viven en hogares conformados por parejas del mismo sexo, tienen el mismo nivel de atención, cuidados, educación, recibiendo la afectividad y la estabilidad emocional que todo niño tiene derecho desde el núcleo familiar. 
Para finalizar, la postura negativa de la adopción de las parejas del mismo sexo es una forma de discriminación y estigmatización de las personas que pertenecen a movimientos o que viven con normalidad su orientación sexual, por lo que es necesario que se hagan las reformas y modificaciones convenientes en los términos expuestos en la figura 1, a fin de que se alcance la igualdad de todas las personas en sus derechos y de esta manera puedan obtener el mismo nivel de reconocimiento que tienen las parejas heterosexuales.

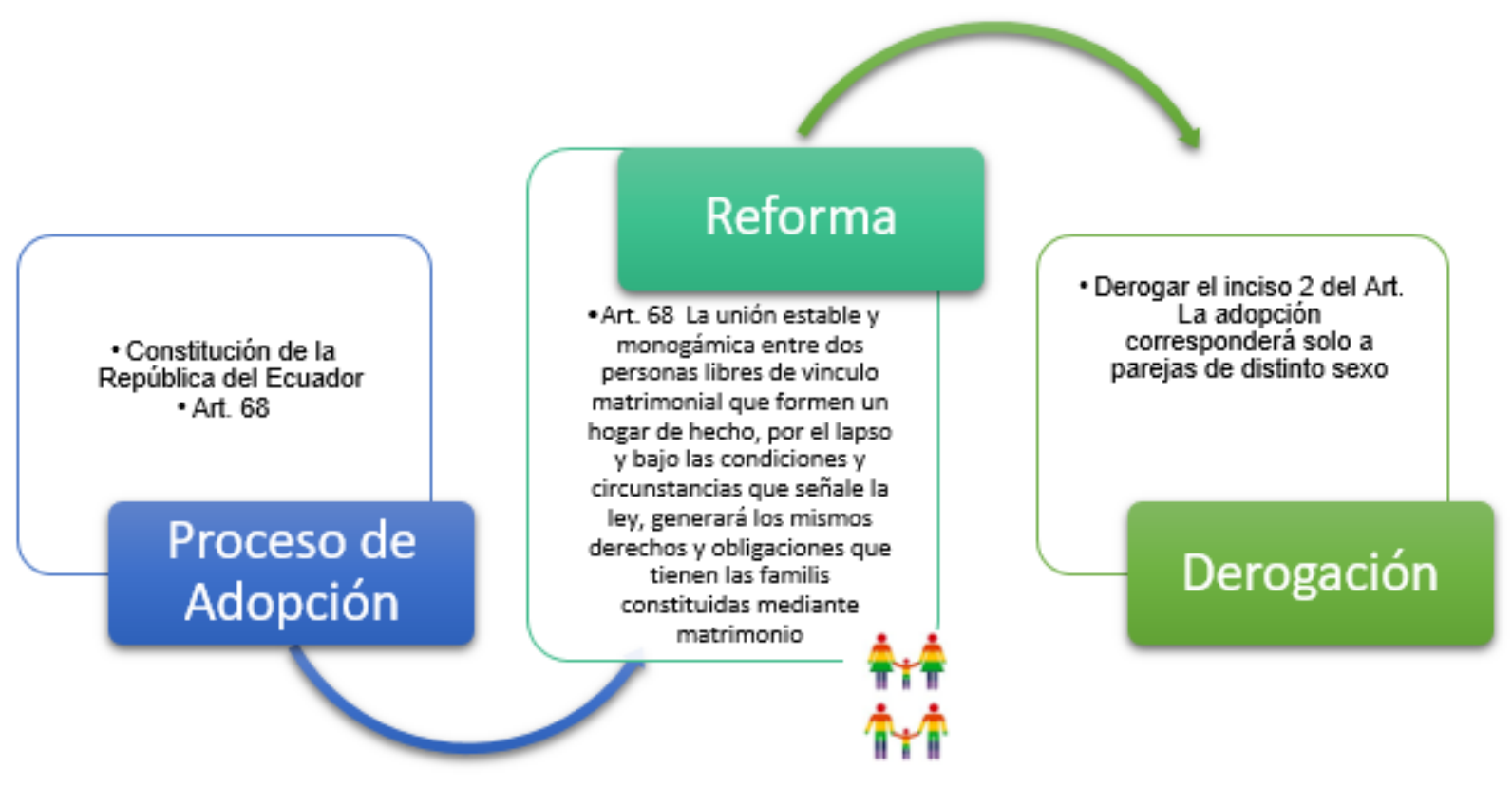

Figura 1. Propuesta de reforma al proceso de adopción 


\section{REFERENCIAS CONSULTADAS}

1. Agrest, B. (2007). Homoparentalidades. Buenos Aires: Nuevas Familias.

2. Asamblea Nacional. (2008). Constitución de la República del Ecuador. Registro Oficial 449 . Quito, Pichincha, Ecuador: Editora Nacional.

3. Asamblea Nacional. (2012). Código Orgánico de la Niñez y Adolescencia. Quito, Pichincha, Ecuador: Corporación de Estudios y Publicaciones.

4. Aylwin, A., y Solar, M. (2012). Trabajo Social Familiar. Santiago de Chile: Editorial Alfaomega.

5. Bolaños, B., Colorado, R., Quintero, J., y Mesa, L. (2019). La homoparentalidad: un interés vigente de la investigación latinoamericana. Repertorio de Medicina y Cirugía, 28(2), 81-90.

6. Bowen, M. (1991). De la Familia al Individuo la diferenciacion del sí mismo en el sistema familiar. Barcelona: Paidos.

7. Campos, A., y Herazo, E. (2015). La adopción por parejas del mismo sexo en Colombia. Revista Colombiana de Psiquiatria, 44(2), 75-76.

8. Caso Bicknell-Rothon en contra del estado ecuatoriano, Sentencia Nro. 184-18SEP-CC (Corte Constitucional del Ecuador 29 de mayo de 2018).

9. CIDH. (2015). Violencia contra personas lesbianas, gays, bisexuales, trans e intersex en América. CIDH. Recuperado de http://www.oas.org/es/cidh/informes/pdfs/ViolenciaPersonasLGBTI.pdf

10. Durán, A. (2012). La Adopción. Revista Jurídica Digital Derecho Ecuador. Quito, Pichincha, Ecuador. Recuperado de http://www.derechoecuador.com/articulos/detalle/archive/doctrinas/derechodelani nezylaadolescencia/2012/01/27/la-adopcion.

11. Gómez, B., Berástegui, A., y Adroher, S. (2015). Se busca familia para un niño. Perspectivas psico-jurídicas sobre la adoptabilidad. Madrid: Dykinson. 
12. González, M. (2015). Trabajo Social y Adopción. Trabajo de Fin de Grado. Jaén, España: Universidad de Jaén. Recuperado de https://hdl.handle.net/10953.1/1684

13. Hernández, A. (1998). Familia, Ciclo Vital y Psicoterapia Sistémica Breve. Santa Fé de Bogotá: El Búho.

14. ONU. (1948). Declaración Universal de los Derechos Humanos. Resolución 217 A (III). París, Francia.

15. ONU. (2007). Principios de Yogyakarta: Principios sobre la aplicación de legislación internacional de los derechos humanos en relación a la orientación sexual y la identidad de género. Organización de las Naciones Unidas.

16. Palacio, M., y Cárdenas, O. (2017). La crisis de la familia: tensión entre lo convencional y lo emergente. Maguaré, 31(1), 43-64.

17. Placeres, J., Olver, D., Rosero, G., y Urgilés J, (2017). La familia homoparental en la realidad y la diversidad familiar actual. Revista Médica Electrónica, 39(2), 361-369. Recuperado http://scielo.sld.cu/scielo.php?script=sci_arttext\&pid=S1684$18242017000200022 \&$ Ing=es\&tlng=pt.

18. Pulecio, M. (2015). Teoría y Práctica de los Principios de Yogyakarta en el derecho internacional de los Derechos Humanos. Revista Análisis Internacional(3), 239-259. Recuperado de https://revistas.utadeo.edu.co/index.php/RAl/article/view/70

19. Quinche, M. (2016). Violencia, omisiones y estructuras que enfrentan las personas LGBTI. Estudios Socio-Jurídicos, 18(2), 49-87. doi:dx.doi.org/10.12804/esj18.02.2016.02

20. Ramírez, A., y Lozada, J. (2017). Entre el estigma homofóbico y el orgullo gay: una aproximación a la historia del activismo LGBTI en Venezuela. Memoria de grado. Mérida, Venezuela: Universidad de Los Andes. Recuperado el 27 de diciembre de 2019, de www.iessdeh.org

21. Rengifo, L. (2016). La adopción homoparental en Colombia: Consideraciones conceptuales y jurisprudenciales. Revista Inciso, 19(2), 1-16. 
22. Rodríguez, A. (2012). Reflexiones sobre la familia y el matrimonio desde la sociología y el derecho como fenómeno no solo social sino también como ciencias. Obtenido de EUMED: www.eumed.net

23. Romo, M. P. (2013). Las familias diversas en la Constitución del Ecuador. Quito: Lecciones básicas de Derecho.

24. Ruiz, S. (2019). Análisis Jurídico de la nueva forma de familia. Tesis de Grado. Riobamba, Ecuador: UNACH. Recuperado de http://dspace.unach.edu.ec/handle/51000/6193

25. Ruiz Armijos, A. J., Narváez Zurita, C. I., \& Erazo Álvarez, J. C. (2019). Tratamiento del impuesto diferido a partir de la valuación de activos biológicos bajo NIIF en la industria camaronera del Ecuador. Koinonía, 310.

26. Sánz, S. (2013). Familia en derecho internacional y europeo. En M. Álvarez, \& R. Cippitani, Diccionario analítico de derechos humanos e integración jurídica. (págs. 251-258). México: Instituto per gli Studi Economici e Giuridici.

27. Véliz Valencia, Y. (2019). Perspectivas de la adopción homoparental en el Ecuador. IUSTITIA SOCIALIS, $4(1), \quad$ 177-192. doi:http://dx.doi.org/10.35381/racii.v4i1.547

28.Zambrano, E. (2017). La familia. Tesis Doctoral. Loja: UTPL. Recuperado de www.dspace.utpl.edu.ec

(C2020 por los autores. Este artículo es de acceso abierto y distribuido según los términos y condiciones de la licencia Creative Commons Atribución-NoComercial-Compartirlgual 4.0 Internacional (CC BY-NC-SA 4.0) (https://creativecommons.org/licenses/by-nc-sa/4.0/). 